\title{
Algebraic Stories from One and from the Other Pockets
}

\author{
Ralf Fröberg $^{1} \cdot$ Samuel Lundqvist $^{1} \cdot$ Alessandro Oneto $^{2,3} \cdot$ Boris Shapiro $^{1}$
}

Received: 7 January 2018 / Revised: 31 May 2018 / Accepted: 18 July 2018 / Published online: 31 July 2018

(c) The Author(s) 2018

\begin{abstract}
In what follows, we present a large number of questions which were posed on the problem solving seminar in algebra at Stockholm University during the period Fall 2014-Spring 2017 along with a number of results related to these problems. Many of the results were obtained by participants of the latter seminar.
\end{abstract}

Keywords Waring problem for forms · Generic and maximal ranks · Ideals of generic forms $\cdot$ Power ideals $\cdot$ Lefschetz properties $\cdot$ Symbolic powers

\section{The Waring Problem for Complex-Valued Forms}

The following famous result on binary forms was proven by Sylvester in 1851. Below we use the terms "forms" and "homogeneous polynomials" as synonyms.

Theorem 1.1 (Sylvester's Theorem, see Sylvester (1973))

To our dear colleague, late Jan-Erik Roos.

The title alludes to the famous collection of mystery novels "Povídky z jedné a z druhé kapsy" by K. Čapek.

Boris Shapiro

shapiro@math.su.se

Ralf Fröberg

ralff@math.su.se

Samuel Lundqvist

samuel@math.su.se

Alessandro Oneto

alessandro.oneto@upc.edu; aless.oneto@gmail.com

1 Department of Mathematics, Stockholm University, 10691 Stockholm, Sweden

2 Department of Mathematics, Universitat Politècnica de Catalunya, Barcelona, Spain

3 Barcelona Graduate School of Mathematics, and Universitat Politècnica de Catalunya, Barcelona, Spain 
(i) A general binary form $f$ of odd degree $k=2 s-1$ with complex coefficients can be written as

$$
f(x, y)=\sum_{j=1}^{s}\left(\alpha_{j} x+\beta_{j} y\right)^{k} .
$$

(ii) A general binary form $f$ of even degree $k=2 s$ with complex coefficients can be written as

$$
f(x, y)=\lambda x^{k}+\sum_{j=1}^{s}\left(\alpha_{j} x+\beta_{j} y\right)^{k} .
$$

Sylvester's result was the starting point of the study of the so-called Waring problem for polynomials which we discuss in this section.

Let $S=\mathbb{C}\left[x_{1}, \ldots, x_{n}\right]$ be the polynomial ring in $n$ variables with complex coefficients. Obviously, $S=\bigoplus_{k>0} S_{k}$ with respect to the standard grading, where $S_{k}$ denotes the vector space of all forms of degree $k$.

Definition 1.2 Let $f$ be a form of degree $k$ in $S$. A presentation of $f$ as a sum of $k$-th powers of linear forms, i.e., $f=\ell_{1}^{k}+\cdots+\ell_{s}^{k}$, where $\ell_{1}, \cdots, \ell_{s} \in S_{1}$, is called a Waring decomposition of $f$. The minimal length of such a decomposition is called the Waring rank of $f$, and we denote it $\operatorname{as} \operatorname{rk}(f)$. By $\operatorname{rk}^{\circ}(k, n)$ we denote the Waring rank of a general complex-valued form of degree $k$ in $n$ variables.

The name of this problem is motivated by its celebrated prototype, i.e., the Waring problem for natural numbers. The latter was posed in 1770 by the British number theorist E. Waring who claimed that, for any positive integer $k$, there exists a minimal number $g(k)$ such that every natural number can be written as a sum of at most $g(k)$ $k$-th powers of positive integers. The famous Lagrange's four-squares Theorem (1770) claims that $g(2)=4$ while the existence of $g(k)$, for any integer $k \geq 2$, is due to D. Hilbert (1900). Exact values of $g(k)$ are currently known only in a few cases although it is generally believed that

$$
g(k)=2 k+\left[(3 / 2)^{k}\right]-2 .
$$

As we mentioned above, the interest in additive decompositions of polynomials goes back to the 19-th century; in the last decades however these types of problems received a lot of additional attention in several areas of pure and applied mathematics. This interest is partially explained by the fact that homogeneous polynomials can be naturally identified with symmetric tensors whose additive structure is important in relation to problems coming from applications (an interested reader can consult Landsberg's book Landsberg (2012)).

The Waring problem for complex-valued forms has three major perspectives: (1) calculation of the rank of a general form, i.e., the rank that occur in a Zariski open (and dense) subset of forms of a given degree; (2) calculation of the maximal rank for forms of a given degree; (3) calculation of the number of minimal decompositions of a given form. In this section, we state some problems related to all three directions. 
Remark 1.3 The Waring problem for polynomials can be studied over other fields as well. However on one hand, over fields of finite characteristic, even Definition 1.2 needs to be clarified; see Gallardo (2000), Car and Gallardo (2004), Car (2008), Gallardo and Vaserstein (2008), Liu and Wooley (2010). On the other hand, the case of real numbers although particularly interesting for applications is also very different from the case of complex numbers from a geometric point of view. In particular, the notion of generic rank has to be refined, since over real numbers Zariski open sets are not necessarily dense; see Comon and Ottaviani (2012), Causa and Re (2011), Blekherman (2015), Bernardi et al. (2017), Michałek et al. (2017), Carlini et al. (2017). Since the situation with these other natural fields is quite different from the case of complex-valued polynomials, we decided not to discuss these questions here.

\subsection{Generic $k$-Rank}

Another motivation for the renewed interest in the Waring problem for polynomials comes from the celebrated result of Alexander and Hirschowitz (1995) which completely describes the Waring $\operatorname{rank}^{\circ} \mathrm{rk}^{\circ}(k, n)$ of general forms of any degree and in any number of variables (it generalizes the above Sylvester's Theorem that claims that the Waring rank of a general binary complex-valued form of degree $k$ equals $\left\lfloor\frac{k}{2}\right\rfloor$ ).

Theorem 1.4 (Alexander-Hirschowitz Theorem, 1995). For all pairs of positive integers $(k, n)$, the generic Waring rank $r k^{\circ}(k, n)$ is given by

$$
r k^{\circ}(k, n)=\left\lceil\frac{\left(\begin{array}{c}
n+k-1 \\
n-1
\end{array}\right)}{n}\right\rceil,
$$

except for the following cases:

(1) $k=2$, where $r k^{\circ}(2, n)=n$;

(2) $k=4, n=3,4,5$; and $k=3, n=5$, where, $r_{g e n}(k, n)$ equals the r.h.s of (1) plus 1 .

Except for sums of powers of linear forms several other types of additive decompositions of homogeneous polynomials have been considered in the literature. These include:

(a) decompositions into sums of completely decomposable forms, i.e., decompositions of the form $f=\sum_{i=1}^{r} \ell_{i, 1} \cdots \ell_{i, d}$; see Arrondo and Bernardi (2011), Shin (2012), Abo (2014), Torrance (2017), Catalisano et al. (2015);

(b) decompositions of the form $f=\sum_{i=1}^{r} \ell_{i, 1}^{d-1} \ell_{i, 2}$; see Catalisano et al. (2002), Ballico (2005), Abo and Vannieuwenhoven (2018);

(c) decompositions of a more general form $f=\sum_{i=1}^{r} \ell_{i, 1}^{d_{1}} \cdots \ell_{i, s}^{d_{s}}$, where $\left(d_{1}, \ldots, d_{s}\right)$ $\vdash d$ any fixed partition of $d$; see Catalisano et al. (2017).

In particular, in Fröberg et al. (2012), Fröberg, Shapiro and Ottaviani considered the following natural generalization of the Waring problem for complex-valued forms (to the best of our knowledge such generalization has not been studied earlier). 
Definition 1.5 Let $k, d$ be positive integers. Given a form $f$ of degree $k d$, a $k$-Waring decomposition is a presentation of $f$ as a sum of $k$-th powers of forms of degree $d$, i.e., $f=g_{1}^{k}+\cdots+g_{s}^{k}$, with $g_{i} \in S_{d}$. The minimal length of such an expression is called the $k$-rank of $f$ and is denoted by $\operatorname{rk}_{k}(f)$. We denote by $\operatorname{rk}_{k}^{\circ}(k d, n)$ the $k$-rank of a general complex-valued form of degree $k d$ in $n$ variables.

In this notation, the case $d=1$ corresponds to the classical Waring problem, i.e., if $k=\operatorname{deg}(f)$, then $\operatorname{rk}(f)=\operatorname{rk}_{k}(f)$ and $\operatorname{rk}^{\circ}(k, n)=\operatorname{rk}_{k}^{\circ}(k, n)$. Since the case $k=1$ is trivial, we assume below that $k \geq 2$.

Problem A Given a triple of positive integers $(k, d, n)$, calculate $r k_{k}^{\circ}(k d, n)$.

The main result of Fröberg et al. (2012) states that, for any triple $(k, d, n)$ as above,

$$
\mathrm{rk}_{k}^{\circ}(k d, n) \leq k^{n-1} \text {. }
$$

At the same time, by a simple parameter count, one has a lower bound for $\operatorname{rk}^{\circ}(k, n)$ given by

$$
\mathrm{rk}_{k}^{\circ}(k d, n) \geq\left\lceil\frac{\left(\begin{array}{c}
n+k d-1 \\
n-1
\end{array}\right)}{\left(\begin{array}{c}
n+d-1 \\
n-1
\end{array}\right)}\right\rceil
$$

A remarkable fact about the upper bound given by (2) is that it is independent of $d$. Therefore, since the right-hand side of (3) equals $k^{n-1}$ when $d \gg 0$, we get that for large values of $d$, the bound in (2) is actually sharp. As a consequence of this remark, for any fixed $n \geq 1$ and $k \geq 2$, there exists a positive integer $d_{k, n}$ such that $\mathrm{rk}_{k}^{\circ}(k d, n)=k^{n-1}$, for all $d \geq d_{k, n}$.

In the case of binary forms, it has been proven that (3) is actually an equality Reznick (2013), Lundqvist et al. (2017). Exact values of $d_{k, n}$, and the behaviour of $\operatorname{rk}_{k}(k d, n)$ for $d \leq d_{k, n}$, have also been computed in the case $k=2$ for $n=3$, 4; see (Lundqvist et al. (2017), Appendix). These results agree with the following conjecture suggested to the authors by Ottaviani in 2014 in a private communication (in the case $k=2$, this conjecture agrees with ([Le et al. (2013), Conjecture 1])).

Conjecture 1.6 The $k$-rank of a general form of degree $k d$ in $n$ variables is given by

$$
r k_{k}^{\circ}(k d, n)= \begin{cases}\min \left\{s \geq 1 \mid s\left(\begin{array}{c}
n+d-1 \\
n-1
\end{array}\right)-\left(\begin{array}{c}
s \\
2
\end{array}\right) \geq\left(\begin{array}{c}
n+2 d-1 \\
n-1
\end{array}\right)\right\}, & \text { for } k=2 ; \\
\min \left\{s \geq 1 \mid s\left(\begin{array}{c}
n+d-1 \\
n-1
\end{array}\right) \geq\left(\begin{array}{c}
n+k d-1 \\
n-1
\end{array}\right)\right\}, & \text { for } k \geq 3 .\end{cases}
$$

Observe that, for $k \geq 3$, Conjecture 1.6 claims that the naïve bound (3) obtained by a parameter count is actually sharp, while, for $k=2$, due to an additional group action there are many defective cases where the inequality (3) is strict. The intuition behind Conjecture 1.6 can be explained by the geometric interpretation of Waring problems in terms of secant varieties, see below.

Remark 1.7 (Waring problems and secant varieties) Problems on additive decompositions, such as all the Waring-type problems mentioned above, can be rephrased geometrically in terms of the so-called secant varieties. Given any projective variety 
$V$, the $s$-secant variety $\sigma_{s}(V)$ is the Zariski closure of the union of all linear spaces spanned by $s$-tuples of points on $V$ (these geometric objects are very classical and have been studied since the beginning of the last century; see e.g. Palatini (1906), Scorza (1908), Palatini (1909), Terracini (1911). An abundance of information on secant varieties can be found in Zak (1993)).

In the case of the classical Waring problem for complex-valued polynomials, we should work with the projective variety parametrized by powers of linear forms in the ambient projective space of homogeneous polynomials in $n$ variables having degree $d$, i.e., our projective variety is the classical Veronese variety $V_{n, d}$. Its $s$-secant variety $\sigma_{s}\left(V_{n, d}\right)$ is the Zariski closure of the set of homogeneous polynomials whose Waring rank is at most $s$. Therefore, the generic Waring $\operatorname{rank} \operatorname{rk}^{\circ}(d, n)$ is the smallest $s$ such that the $s$-secant variety coincides with the whole space of forms of degree $d$. Since the dimension of the affine cone over the Veronese variety $V_{n, d}$ has dimension $n$ and the dimension of its ambient space is $\left(\begin{array}{c}n+d \\ n\end{array}\right)$, Alexander-Hirschowitz Theorem claims that the $s$-secant variety $\sigma_{s}\left(V_{n, d}\right)$ has the expected dimension, which is equal to $s n$, except for a few defective cases, where the dimension is strictly smaller than the expected one.

In the case of $k$-Waring decompositions, we consider the variety of powers $V_{n, d}^{(k)}$, i.e., the variety of $k$-th powers of forms of degree $d$ in the ambient space of forms of degree $k d$. Hence, Problem A can be rephrased as a problem about the dimensions of secant varieties for $V_{n, d}^{(k)}$. The dimension of the affine cone over the variety of powers $V_{n, d}^{(k)}$ is $\left(\begin{array}{c}n+d-1 \\ n-1\end{array}\right)$ and the dimension of its ambient space is $\left(\begin{array}{c}n+k d-1 \\ n-1\end{array}\right)$; hence, Conjecture 1.6 claims that, for $k \geq 3$, the affine cone over the $s$-secant variety has the expected dimension, which is $s\left(\begin{array}{c}n+k d-1 \\ n-1\end{array}\right)$. The case $k=2$ is intrinsically pathological and gives defective cases for an arbitrary pair $(d, n)$ which is similar to the case (1) of Theorem 1.4; see [Oneto (2016), Remark 3.3.5] (we refer to Lundqvist et al. (2017) for more details about this geometric interpretation of Problem A).

\subsection{Maximal $k$-Rank}

A harder problem, which is largely open even in the classical case of Waring decompositions, deals with the computation of the maximum of $k$-ranks taken over all complex-valued forms of degree divisible by $k$.

Definition 1.8 Given a triple $(k, d, n)$, denote by $\operatorname{rk}_{k}^{\max }(k d, n)$ the minimal number of terms such that every form of degree $k d$ in $n+1$ variables can be represented as the sum of at most $\mathrm{rk}_{k}^{\max }(k d, n) k$-th powers of forms of degree $d$. The number $\mathrm{rk}_{k}^{\max }(k d, n)$ is called the maximal $k$-rank. Similarly to the above, we omit the subscript when considering the classical Waring rank, i.e., for $d=1$.

In [Reznick (2013), Theorem 5.4], Reznick shows that the maximal Waring rank of binary forms of degree $k$, which is equal to $k$, is attained exactly on the binary forms representable as $\ell_{1} \ell_{2}^{k-1}$, where $\ell_{1}$ and $\ell_{2}$ are any two non-proportional linear binary forms. As the author says, the latter result "must be ancient", but we could not find a suitable reference prior to Reznick (2013).

Problem B Given a triple of positive integers $(k, d, n)$, calculate $r k_{k}^{\max }(k d, n)$. 
At the moment, we have an explicit conjecture about the maximal $k$-rank of forms of degree $k d$ only in the case of binary forms.

Conjecture 1.9 For any positive integers $k$ and $d$, the maximal $k$-rank $r k_{k}^{\max }(k d, 2)$ of binary forms is $k$. Additionally, in the above notation, binary forms representable by $\ell_{1} \ell_{2}^{k d-1}$, where $\ell_{1}$ and $\ell_{2}$ are non-proportional linear forms, have the latter maximal $k$-rank.

Conjecture 1.9 is obvious for $k=2$ since, for any binary form $f$ of degree $2 d$, we can write

$$
f=g_{1} g_{2}=\left(\frac{1}{2}\left(g_{1}+g_{2}\right)\right)^{2}+\left(\frac{i}{2}\left(g_{1}-g_{2}\right)\right)^{2}, \quad \text { with } g_{1}, g_{2} \in S_{d} \text {. }
$$

The first non-trivial case is that of binary sextics, i.e., $k=3, d=2$, which has been settled in Lundqvist et al. (2017) where it has also been shown that the 4-rank of $x_{1} x_{2}^{7}$ is equal to 4 .

The best known general result about maximal ranks is due to Bleckherman and Teitler, see Blekherman and Teitler (2015), where they prove that the maximal rank is always at most twice as big as the generic rank. This fact is true for any additive decomposition and, in particular, both for the classical $(d=1)$ and for the higher $(d \geq 2)$ Waring ranks. In the classical case of Waring ranks, the latter bound is (almost) sharp for binary forms, but in many other cases it seems rather crude. At present, better bounds are known only in few special cases of low degrees Ballico and Paris (2017), Jelisiejew (2014). To the best of our knowledge, the exact values of the maximal Waring rank are only known for binary forms (classical, see Reznick (2013)), quadrics (classical), ternary cubics [see Segre (1942), Landsberg and Teitler (2010)], ternary quartics, see Kleppe (1999), ternary quintics, see De Paris (2015) and quaternary cubics, see Segre (1942).

\subsection{The $k$-Rank of Monomials}

Let $m=x_{1}^{a_{1}} \cdots x_{n}^{a_{n}}$ be a monomial with $0<a_{1} \leq a_{2} \leq \cdots \leq a_{n}$. It has been shown in Carlini et al. (2012) that the classical Waring rank of $m$ is equal to $\frac{1}{\left(a_{1}+1\right)} \prod_{i=1, \ldots, n}\left(a_{i}+\right.$ 1).

Problem $C$ Given $k \geq 3$ and a monomial $m$ of degree $k d$, determine the monomial $k$-rank $r k_{k}(m)$.

E. Carlini and A. Oneto settled the case of the 2-rank, see Carlini and Oneto (2015). Namely, if $m$ is a monomial of degree $2 d$, then we can write $m=m_{1} m_{2}$, where $m_{1}$ and $m_{2}$ are monomials of degree $d$. From identity (5), it follows that the 2-rank of $m$ is at most two. On the other hand, $m$ has rank one exactly when we can choose $m_{1}=m_{2}$, i.e., when the power of each variable in $m$ is even. In Carlini and Oneto (2015), also the case $k=3$ in two and three variables has been settled, but, in general, for $k \geq 3$, the question about the $k$-rank of monomials of degree $k d$, is still open. At present, we are only aware of only two general results in this direction. Namely, Carlini and 
Oneto (2015) contains the bound $\mathrm{rk}_{k}(m) \leq 2^{k-1}$, and recently, S. Lundqvist, A. Oneto, B. Reznick, and B. Shapiro have shown that $\operatorname{rk}_{k}(m) \leq k$ when $d \geq n(k-2)$, see Lundqvist et al. (2017). Thus, for fixed $k$ and $n$, all but a finite number of monomials of degree divisible by $k$ have $k$-rank less than $k$.

In the case of binary forms, a bit more is currently known which motivates the following question.

Problem D Given $k \geq 3$ and a monomial $x^{a} y^{b}$ of degree $a+b=k d$, it is known that $r k_{k}\left(x^{a} y^{b}\right) \leq \max (s, t)+1$, where $s$ and $t$ are the remainders of the division of $a$ and $b$ by $k$, see Carlini and Oneto (2015). Is it true that the latter inequality is, in fact, an equality?

The latter question has positive answer for $k=2$ (see [Carlini and Oneto (2015), Theorem 3.2]) and $k=3$ (see [Carlini and Oneto (2015), Corollary 3.6]). For $k=4$, we know that $x y^{7}$ has 4-rank equal to 4 (see [Lundqvist et al. (2017), Example 4.7]). As far as we know, these are the only known cases.

\subsection{Degree of the Waring Map}

Here again, we concentrate on the case of binary forms (i.e., $n=2$ ). As we mentioned above, in this case, it is proven that

$$
\mathrm{rk}_{k}^{\circ}(k d, 2)=\left\lceil\frac{\operatorname{dim} S_{k d}}{\operatorname{dim} S_{d}}\right\rceil=\left\lceil\frac{k d+1}{d+1}\right\rceil .
$$

Definition 1.10 We say that a pair $(k, d)$ is perfect if $\frac{k d+1}{d+1}$ is an integer.

All perfect pairs are easy to describe.

Lemma 1.11 The set of all pairs $(k, d)$ for which $\frac{k d+1}{d+1} \in \mathbb{N}$ splits into the disjoint sequences $E_{j}:=\{(j d+j+1, d) \mid d=1,2, \ldots\}$. In each $E_{j}$, the corresponding quotient equals $j d+1$.

Given a perfect pair $(k, d)$, set $s:=\frac{k d+1}{d+1}$. Consider the map

$$
W_{k, d}: S_{d} \times \ldots \times S_{d} \rightarrow S_{k d}, \quad\left(g_{1}, \ldots, g_{s}\right) \mapsto g_{1}^{k}+\ldots+g_{s}^{k}
$$

Let $\widetilde{W}_{k, d}$ be the same map, but defined up to a permutation of the $g_{i}$ 's. We call it the Waring map. By [Lundqvist et al. (2017), Theorem 2.3], $\widetilde{W}_{k, d}$ is a generically finite map of complex linear spaces of the same dimension. By definition, its degree is the cardinality of the inverse image of a generic form in $S_{k d}$.

Problem E Calculate the degree of $\widetilde{W}_{k, d}$ for perfect pairs $(k, d)$.

For the classical Waring decomposition (i.e., for $d=1$ ), we get a perfect pair if and only if $k$ is odd. From Sylvester's Theorem (Theorem 1.1), we know that in this case the degree of the Waring map is 1, i.e., the general binary form of odd degree has a unique Waring decomposition, up to a permutation of its summands. 
Remark 1.12 For the classical Waring decomposition, the problem of counting the number of decompositions of a generic form of degree $k$ in $n$ variables under the assumption that $\frac{\left(\begin{array}{c}n+k-1 \\ n-1\end{array}\right)}{n}$ is an integer which coincides with the corresponding generic Waring rank, has been actively studied not just fro binary forms but also in the case of many variables. In modern terminology, the cases when the general form of a given degree has a unique decomposition, up to a permutation of the summands, are called identifiable. Besides the above mentioned case of binary forms of odd degree, some other identifiable cases are known. These are the quaternary cubics (Sylvester's Pentahedral Theorem, see Sylvester (1973)) and the ternary quintics Hilbert (1888b), Palatini (1903), Richmond (1904), Massarenti and Mella (2013). Recently, F. Galuppi and M. Mella proved that these are the only possible identifiable cases, Galuppi and Mella (2017). (We refer to Chiantini et al. (2017) for the current status of this problem.)

\section{Ideals of Generic Forms}

Let $I$ be a homogeneous ideal in $S$, i.e., an ideal generated by homogeneous polynomials. The ideal $I$ and the quotient algebra $R=S / I$ inherit the grading of the polynomial ring.

Definition 2.1 Given a homogeneous ideal $I \subset S$, we call the function

$$
\operatorname{HF}_{R}(i):=\operatorname{dim}_{\mathbb{C}} R_{i}=\operatorname{dim}_{\mathbb{C}} S_{i}-\operatorname{dim}_{\mathbb{C}} I_{i}
$$

the Hilbert function of $R$. The power series

$$
\operatorname{HS}_{R}(t):=\sum_{i \in \mathbb{N}} \operatorname{HF}_{R}(i) t^{i} \in \mathbb{C}[[t]]
$$

is called the Hilbert series of $R$.

Let $I$ be a homogeneous ideal generated by forms $f_{1}, \ldots, f_{r}$ of degrees $d_{1}, \ldots, d_{r}$, respectively. It was shown in Fröberg and Löfwall (1990) that, for fixed parameters $\left(n, d_{1}, \ldots, d_{r}\right)$, there exists only a finite number of possible Hilbert series for $S / I$, and that there is a Zariski open subset in the space of coefficients of the $f_{i}$ 's on which the Hilbert series of $S / I$ is one and the same. Additionally, in the appropriate sense, it is the minimal series among all possible Hilbert series, see below. We call algebras with this Hilbert series generic. There is a longstanding conjecture describing this minimal Hilbert series due to the first author, see Fröberg (1985).

Conjecture 2.2 (Fröberg's Conjecture, 1985). Let $f_{1}, \ldots, f_{r}$ be generic forms of degrees $d_{1}, \ldots, d_{r}$, respectively. Then the Hilbert series of the quotient algebra $R=S /\left(f_{1}, \ldots, f_{r}\right)$ is given by

$$
\mathrm{HF}_{R}(t)=\left[\frac{\prod_{i=1}^{r}\left(1-t^{d_{i}}\right)}{(1-t)^{n}}\right]_{+} .
$$


Here $\left[\sum_{i \geq 0} a_{i} z^{i}\right]_{+}:=\sum_{i \geq 0} b_{i} z^{i}$, with $b_{i}=a_{i}$ if $a_{j} \geq 0$ for all $j \leq i$ and $b_{i}=$ 0 otherwise. In other words, $\left[\sum_{i \geq 0} a_{i} z^{i}\right]_{+}$is the truncation of a real power series $\sum_{i \geq 0} a_{i} z^{i}$ at its first non-positive coefficient.

Conjecture 2.2 has been proven in the following cases: for $r \leq n$ (easy exercise, since in this case $I$ is a complete intersection); for $n \leq 2$, Fröberg (1985); for $n=3$, Anick (1986), for $r=n+1$, which follows from Stanley (1978). Additionally, in Hochster and Laksov (1987) it has been proven that (6) is correct in the first nontrivial degree $\min _{i=1}^{r}\left(d_{i}+1\right)$. There are also other special results in the case $d_{1}=\cdots=d_{r}$, see Fröberg and Hollman (1994), Aubry (1995), Migliore and Miro-Roig (2003), Nicklasson (2017a), Nenashev (2017).

We should also mention that Fröberg and Lundqvist (2018) contains a survey of the existing results on the generic series for various algebras and it also studies the (opposite) problem of finding the maximal Hilbert series for fixed parameters $\left(n, d_{1}, \ldots, d_{r}\right)$.

It is known that the actual Hilbert series of the quotient ring of any ideal with the same numerical parameters is lexicographically larger than or equal to the conjectured one. This fact implies that if for a given discrete data $\left(n, d_{1}, \ldots, d_{r}\right)$, one finds just a single example of an algebra with the Hilbert series as in (6), then Conjecture 2.2 is settled in this case.

Although algebras with the minimal Hilbert series constitute a Zariski open set, they are hard to find constructively. We are only aware of two explicit constructions giving the minimal series in the special case $r=n+1$, namely $\mathrm{R}$. Stanley's choice $x_{1}^{d_{1}}, \ldots, x_{n}^{d_{n}},\left(x_{1}+\cdots+x_{n}\right)^{d_{n+1}}$, and C. Gottlieb's choice $x_{1}^{d_{1}}, \ldots, x_{n}^{d_{n}}, h_{d_{n+1}}$, where $h_{d}$ denotes the complete homogeneous symmetric polynomial of degree $d$, (private communication). To the best of our knowledge, already in the next case $r=n+2$ there is no concrete guess about how to construct a similar example. There is however a substantial computer-based evidence pointing towards the possibility of replacing generic forms of degree $d$ by a product of generic forms of much smaller degrees. We present some problems and conjectures related to such pseudo-concrete constructions below.

\subsection{Hilbert Series of Generic Power Ideals}

Differently from the situation occurring in Stanley's result, for ideals generated by more than $n+1$ powers of generic linear forms, there are known examples of $\left(n, d_{1}, \ldots, d_{r}\right)$ for which algebras generated by powers of generic linear forms fail to have the Hilbert series as in (6).

Recall that ideals generated by powers of linear forms are usually referred to as power ideals. Due to their appearance in several areas of algebraic geometry, commutative algebra and combinatorics, they have been studied more thoroughly than generic ideals. In the next section, we will discuss their relation with the so-called fat points. (For a more extensive survey on power ideals, we refer to the nice paper by Ardila and Postnikov (2010), Ardila and Postnikov (2015).) 
Studying Hilbert functions of generic power ideals, Iarrobino formulated the following conjecture, usually referred to as the Fröberg-Iarrobino Conjecture, see Iarrobino (1997), Chandler (2005).

Conjecture 2.3 (Fröberg-Iarrobino Conjecture). Given generic linearforms $\ell_{1}, \ldots, \ell_{r}$ and a positive integer $d$, let $I$ be the power ideal generated by $\ell_{1}^{d}, \ldots, \ell_{r}^{d}$. Then the Hilbert function of $R=S / I$ coincides with that in (6), except for the cases $(n, r)=(3,7),(3,8),(4,9),(5,14)$ and possibly for $r=n+2$ and $r=n+3$.

This conjecture is still largely open. In Fröberg and Hollman (1994), Fröberg and Hollman checked it for low degrees and small number of variables using the first version of the software package Macaulay2, see Grayson and Stillman (2002). In the last decades, some progress has been made in reformulation of Conjecture 2.3 in terms of the ideals of fat points and linear systems. We will return to this topic in the next section.

\subsection{Hilbert series of other classes of ideals}

Computer experiments suggest that in order to always generically get the Hilbert function as in (6) we need to replace power ideals by slightly less special ideals.

For example, given a partition $\mu=\left(\mu_{1}, \ldots, \mu_{t}\right) \vdash d$, we call by a $\mu$-power ideal an ideal generated by forms of the type $\left(\mathbf{l}_{1}^{\mu}, \ldots, \mathbf{l}_{r}^{\mu}\right)$, where $\mathbf{l}_{i}^{\mu}=\ell_{i, 1}^{\mu_{1}} \cdots \ell_{i, t}^{\mu_{t}}$ and $\ell_{i, j}$ 's are distinct linear forms.

Problem F For $\mu \neq(d)$, does a generic $\mu$-power ideal have the same Hilbert function as in (6)?

Computer computations of the Hilbert series of $\mu$-power ideals whose generators are produced randomly suggest a positive answer to the latter problem (such calculations can be preformed in any computer algebra software such as $\operatorname{CoCoA}$, see Abbott and Bigatti (2014), Macaulay2, see Grayson and Stillman (2002) or Singular, see Decker et al. (2018)). Nicklasson has also conjectured that ideals generated by powers of generic forms of degree $\geq 2$ have the Hilbert series as in (6).

Conjecture 2.4 (Nicklasson (2017a)). For generic forms $g_{1}, \ldots, g_{r}$ in $n$ variables and of degree $d>1$, the ideal $\left(g_{1}^{k}, \ldots, g_{r}^{k}\right)$ has the Hilbert series equal to

$$
\left[\frac{\left(1-t^{d k}\right)^{r}}{(1-t)^{n}}\right]_{+} .
$$

Remark 2.5 (Power ideals and secant varieties). In Remark 1.7, we explained the relation between Waring problems and the study of dimensions of secant varieties. Here, we want to relate the computation of dimensions of secant varieties with the study of Hilbert functions of the families of ideals we just presented.

The standard way to compute the dimension of a variety $V$ is to consider its tangent space at a generic smooth point. In Terracini (1911), A. Terracini proved the so-called Terracini Lemma which claims that the tangent space at a generic smooth point of 
the $s$-secant variety $\sigma_{s}(V)$ coincides with the linear span of the tangent spaces at $s$ generic smooth points of the variety $V$. Now, if we consider the Veronese variety $V_{n, d}$ of $d$-th powers of linear forms, it is easy to observe that the affine cone over the tangent space at a point $\left[\ell^{d}\right] \in V_{n, d}$ coincides with the $n$-dimensional linear space $\left\{\left[\ell^{d-1} m\right] \mid m\right.$ is any linear form $\}$. Therefore, the dimension of the affine cone over the tangent space to the $s$-secant variety of $V_{n, d}$ at a generic point of the linear span $\left\langle\left[\ell_{1}^{d}\right], \ldots,\left[\ell_{s}^{d}\right]\right\rangle$, where the $\ell_{i}$ 's are generic linear forms, is equal to the dimension of the homogeneous part of degree $d$ of the ideal $\left(\ell_{1}^{d-1}, \ldots, \ell_{s}^{d-1}\right)$. Hence, the defective cases listed in Alexander-Hirschowitz Theorem (Theorem 1.4) can be described in terms of the defective cases listed in the Fröberg-Iarrobino's Conjecture.

In a similar way, the tangent space at a point $\left[g^{k}\right]$ lying on the variety of powers $V_{n, d}^{(k)}$ is given by the $n$-dimensional linear space $\left\{\left[g^{k-1} m\right] \mid m\right.$ is any linear form $\}$. Therefore by Terracini's Lemma we can relate Conjecture 2.4 with the computation of the dimensions of secant varieties to varieties of powers and, therefore, to generic $k$-ranks. In particular, in [Lundqvist et al. (2017), Theorem A.3], the authors proved that Conjecture 2.4 implies Conjecture 1.6 about generic $k$-ranks. In [Lundqvist et al. (2017), Lemma 2.2], it was also shown that Conjecture 2.4 holds in the case of binary forms. The proof is obtained by specializing each one of the $g_{i}$ 's to be the $d$-th power of a generic linear form and applying the fact that generic power ideals in two variables have the generic Hilbert series Geramita and Schenck (1998). It is worth to mention that the same idea as in [Lundqvist et al. (2017), Lemma 2.2] gives a positive answer to Problem $\mathrm{F}$ in the case of binary forms, by specializing $\ell_{i, 1}=\ldots=\ell_{i, t}$, for $i=1, \ldots, r$.

\subsection{Lefschetz Properties of Graded Algebras}

We say that a graded algebra $A$ has the weak Lefschetz property (WLP) if there exists a linear form $\ell$ such that for all $i$, the multiplication map $\times \ell: A_{i} \rightarrow A_{i+1}$ has maximal rank, i.e., is either injective or surjective. Similarly, we say that $A$ has the strong Lefschetz property (SLP) if there exists a linear form $\ell$ such that for all $i$ and $k$, the map $\times \ell^{k}: A_{i} \rightarrow A_{i+k}$ has maximal rank.

Being tightly connected to many branches of mathematics, the Lefschetz properties of graded algebras has evolved into an important area of research in commutative algebra. Here we concentrate on problems related to complete intersections and generic forms. For more references and open problems related to the Lefschetz properties, we refer to Migliore and Nagel (2013), Michałek and Miro-Roig (2016), Migliore et al. (2017), and Harima et al. (2013).

Stanley's proof of the Fröberg conjecture for $r=n+1$ is actually also a proof of the fact that every monomial complete intersection has the SLP. The proof uses the hard Lefschetz theorem, but other proofs has since then been given. An elementary ring theoretic proof appeared in Reid et al. (1991). In the same paper, it was conjectured that each complete intersection has the WLP and the SLP. The conjecture also appears as a question in Migliore and Nagel (2013). The conjecture is true in three variables, see Harima et al. (2003), but remains open in four or more variables. The next problem originally appeared in an unpublished paper by J. Herzog and D. Popescu, see Herzog and Popescu (2005). 
Problem G Let $f_{1}, \ldots, f_{r}$ be generic forms in $S$, and $r \geq n$. Does $S /\left(f_{1}, \ldots, f_{r}\right)$ have the SLP?

Remark 2.6 The answer to Problem G in the case $r=n$ is "Yes", and follows from Stanley's result and semicontinuity.

In Boij et al. (2018), the Lefschetz properties for powers of monomial complete intersections are studied. For the ring $T_{n, d, k}=S /\left(x_{1}^{d}, \ldots, x_{n}^{d}\right)^{k}$, it is shown that for $k \geq d^{n-2}, n \geq 3,(n, d) \neq(3,2), T_{n, d, k}$ fails the WLP. For $n=3$, there is an explicit conjecture for when the WLP holds. Additionally, there is some information about $n>3$. It is shown that $T_{n, 2,2}$ satisfies the WLP when $n$ is odd, and it is believed that $T_{n, 2,2}$ has the WLP also for $n$ even. When $n \geq 11$, computer studies suggest that $T_{n, d, k}$ fails the WLP when $(d, k) \notin\{(2,2),(2,3),(3,2)\}$.

Problem $\mathrm{H}$ When are the WLP and the SLP true for $T_{n, d, k}$ ?

In characteristic $p$, not every monomial complete intersection satisfies the WLP. There is a complete classification of the monomial complete intersections of uniform degrees that enjoy the WLP, see Brenner and Kaid (2011) $(n=3)$ and Kustin and Vraciu (2014) ( $n \geq 4)$. The SLP has been classified also for mixed degrees, see Nicklasson (2017b) $(n=2)$ and Lundqvist and Nicklasson (2016) $(n \geq 3)$. However, it remains an open problem to classify the monomial complete intersections of mixed degrees which has the WLP. Partial results appear in Vraciu (2015) and Lundqvist and Nicklasson (2016).

Problem I In characteristic p, classify the monomial complete intersections (of mixed degree) which has the WLP.

Let us now introduce the concept of the $\mu$-Lefschetz properties, where $\mu=$ $\left(\mu_{1}, \ldots, \mu_{k}\right)$ is a partition of $d$, i.e., $\sum_{i=1}^{k} \mu_{i}=d$. We say that an algebra has the $\mu$-Lefschetz property if $\times \mathbf{l}^{\mu}: A_{i} \rightarrow A_{i+d}$ has maximal rank for all $i$, where $\mathbf{l}^{\mu}=\ell_{1}^{\mu_{1}} \cdots \ell_{k}^{\mu_{k}}$, and $\ell_{i}$ 's are generic linear forms.

Problem J For $R=S /\left(f_{1}, \ldots, f_{r}\right)$, where $f_{1}, \ldots, f_{r}$ are generic forms, does $R$ satisfy the $\mu$-Lefschetz property for all partitions $\mu$ ?

Remark 2.7 (Lefschetz properties and Fröberg's conjecture). The study of Lefschetz properties is relevant for our problems about the Hilbert functions of particular classes of ideals and Fröberg's Conjecture. Indeed, if $I$ is an ideal having the Hilbert function as in (6) and if the map $\times f: A_{i} \rightarrow A_{i+d}$ of multiplication by a form $f$ of degree $d$ has maximal rank for any $i$, then, we can conclude that the ideal $I+(f)$ also has the Hilbert function as in (6). Therefore, the study of the aforementioned problems about Lefschetz properties might allow to compute Hilbert functions "inductively", by adding one generator at a time. Notice also that replacing SLP by WLP in Problem $\mathrm{G}$ gives a special case of the Fröberg conjecture.

\section{Symbolic Powers}

For a prime ideal $\wp$ in a Noetherian ring $R$, define its $m$-th symbolic power $\wp^{(m)}$ as

$$
\wp^{(m)}=\wp^{m} R_{\wp} \cap R .
$$


It is the $\wp$-primary component of $\wp^{m}$. For a general ideal $I$ in $R$, its $m$-th symbolic power is defined as $I^{(m)}=\bigcap_{\wp \in \operatorname{Ass}(I)}\left(I^{m} R_{\wp} \cap R\right)$, where Ass $(I)$ is the set of associated primes of $I$.

\subsection{Hilbert Functions of Fat Points}

Let $I_{X}$ be the ideal in $\mathbb{C}\left[x_{1}, \ldots, x_{n}\right]$ defining a scheme of reduced points $X=P_{1}+$ $\ldots+P_{s}$ in $\mathbb{P}^{n-1}$, say $I_{X}=\wp_{1} \cap \cdots \cap \wp_{s}$ where $\wp_{i}$ is the prime ideal defining the point $P_{i}$. Then, the $m$-th symbolic power $I^{(m)}$ is the ideal $I_{X}^{(m)}=\wp_{1}^{m} \cap \cdots \cap \wp_{s}^{m}$ which defines the scheme of $m$-fat points $m X=m P_{1}+\cdots+m P_{s}$.

Ideals of 0 -dimensional schemes have been studied since the beginning of the last century. Their Hilbert functions are of particular interest since they are related to polynomial interpolation problems. Indeed, the homogeneous part of degree $d$ of the ideal $I_{X}^{(m)}$ is the space of hypersurfaces of degree $d$ in $\mathbb{P}^{n-1}$ passing through each $P_{i}$ to order at least $m-1$, i.e., it is the space of polynomials of degree $d$ whose partial differentials up to order $m-1$ vanish at every $P_{i}$. In a more geometric language, this is the linear system of hypersurfaces of degree $d$ in $\mathbb{P}^{n-1}$ having multiple base points of multiplicity $m$ at the support of $X$.

It is well-known that the Hilbert function of a 0 -dimensional scheme is strictly increasing until it reaches the multiplicity of the scheme, see [Iarrobino (2006), Theorem 1.69]. Hence, since the degree of an $m$-fat point in $\mathbb{P}^{n-1}$ is $\left(\begin{array}{c}n-1+m-1 \\ n-1\end{array}\right)$, the expected Hilbert function is

$$
\exp \cdot \mathrm{HF}_{S / I_{X}^{(m)}}(d)=\min \left\{\left(\begin{array}{c}
n-1+d \\
n-1
\end{array}\right), s\left(\begin{array}{c}
n-1+m-1 \\
n-1
\end{array}\right)\right\} .
$$

In the case of simple generic points $X=P_{1}+\cdots+P_{s}$, i.e., for $m=1$, it is known that the actual Hilbert function is as expected. Indeed, the homogeneous part of $I_{X}$ in degree $d$ is obtained by solving a system of linear equations defined by the matrix $\left(m_{i}\left(P_{j}\right)\right)_{i j}$, where the set of $m_{i}$ 's forms a standard monomial basis for the vector space of homogeneous polynomials of degree $d$ and the symbol $m_{i}\left(P_{j}\right)$ denotes the evaluation of the monomial at the point. If the points are generic, the latter matrix has maximal rank; see Geramita and Orecchia (1981).

In the case of double points $(m=2)$, counterexamples to the latter statement were known since the end of the 19-th century. In 1995, after a series of important papers, Alexander and Hirschowitz proved that the classically known counterexamples were the only ones, see Theorem 1.4. For higher multiplicity, very little information is available at present. In the case of the projective plane, a series of equivalent conjectures have been formulated by Segre (1961), Harbourne (1986), Gimigliano (1987) and Hirschowitz (1989). They are baptized as the SHGH-Conjecture, see Harbourne (2000) for a survey of this topic.

Apolarity Theory is a very useful tool in studying the ideals of fat points and it ties together all the algebraic stories we have mentioned above. In particular, the following lemma is crucial (we refer to Iarrobino (2006) and Geramita (1996) for an extensive description of this issue). 
Lemma 3.1 (Apolarity Lemma) Let $X=P_{1}+\cdots+P_{s}$ be a scheme of reduced points in $\mathbb{P}^{n-1}$ and let $L_{1}, \ldots, L_{s}$ be linear forms in $\mathbb{C}\left[x_{0}, \ldots, x_{n}\right]$ such that, for any $i$, the coordinates of $P_{i}$ are the coefficients of $\ell_{i}$. Then, for every $m \leq d$,

$$
\mathrm{HF}_{S / I_{X}^{(m)}}(d)=\operatorname{dim}_{\mathbb{C}}\left[\left(\ell_{1}^{d-m+1}, \ldots, \ell_{s}^{d-m+1}\right)\right]_{d} .
$$

Using this statement we obtain that the calculation of the Hilbert function of a scheme of fat points is equivalent to the calculation of the Hilbert function of the corresponding power ideal. In particular, the Fröberg-Iarrobino Conjecture (Conjecture 2.3) can be rephrased as a conjecture about the Hilbert function of ideals of generic fat points.

Remark 3.2 (Fat points and secant varieties). In Remark 2.5, we described the relation of the study of the Hilbert functions for power ideals with the study of the dimensions of the secant varieties of Veronese varieties and with the problem of calculation of generic Waring ranks. By Apolarity Lemma, we can observe that the study of the Hilbert functions of power ideals is directly related to the computation of the Hilbert functions for fat points. In particular, the aforementioned theorem of Alexander and Hirschowitz (Theorem 1.4) is in fact a result about the Hilbert function of double points in generic position which implies the results on the dimensions of the secant varieties of Veronese varieties and generic Waring ranks.

We have also seen how generic $k$-ranks are related to the dimensions of the secant varieties of varieties of powers and to the Hilbert functions of ideals generated by powers of forms of degree higher than one. Unfortunately, as far as we know, there is no appropriate version of Apolarity Lemma in such a setting. For this reason, most of the classical approaches to Waring problems do not apply in the case of $k$-ranks and new ideas are required.

It is worth mentioning that not only the Hilbert functions of double points in projective spaces are related to secant varieties of particular projective varieties and additive decomposition problems. In the study of the dimensions of secant varieties of several classical varieties there appear other 0-dimensional schemes whose Hilbert functions are important. For example, to compute the dimensions of the secant varieties of tangential varieties of Veronese varieties which are closely related to decompositions of the form $\sum_{i=1}^{r} \ell_{i, 1}^{d-1} \ell_{i, 2}$, one has to study the Hilbert functions of the unions of $(3,2)$-points, where a $(3,2)$-point is by definition a 0 -dimensional scheme obtained by intersecting a 3 -fat point with a 2 -fat line passing through it, i.e., it is the 0 -dimensional scheme defined by an ideal of the type $I_{P}^{3}+I_{L}^{2}$, where $L$ is a line passing through the point $P$; see Bernardi et al. (2009).

Another question posed by R. Fröberg during the problem solving seminars at Stockholm University was about the ideals of generic fat points in a multi-graded space. A point in a multi-projective space $P \in \mathbb{P}^{n_{1}-1} \times \ldots \times \mathbb{P}^{n_{t}-1}$ is defined by a prime ideal $\wp$ in the multi-graded polynomial ring $S=\mathbb{C}\left[x_{1,1}, \ldots, x_{1, n_{1}} ; \ldots ; x_{t, 1}, \ldots, x_{t, n_{t}}\right]=$ $\bigoplus_{I \subset \mathbb{N}^{t}} S_{I}$, where $S_{I}$ is the vector space of multi-graded polynomials of multi-degree $I=\left(i_{1}, \ldots, i_{t}\right) \in \mathbb{N}^{t}$. A scheme of fat points $m X=m P_{1}+\cdots+m P_{s}$ is the scheme associated with the multi-graded ideal $\wp_{1}^{m} \cap \cdots \cap \wp_{s}^{m}$. 
Problem K Given a scheme of generic fat points $m X \subset \mathbb{P}^{n_{1}-1} \times \cdots \times \mathbb{P}^{n_{t}-1}$, what is the multi-graded Hilbert function $\operatorname{HF}_{S / I_{m X}}(I)$, for $I \in \mathbb{N}^{t}$ ?

This question was first considered by Catalisano et al. (2005) who solved it in the case of double points, i.e., for $m=2$, in any $\mathbb{P}^{1} \times \cdots \times \mathbb{P}^{1}$. Recently, A. Oneto jointly with Carlini and Catalisano solved the case of triple points $(m=3)$ in $\mathbb{P}^{1} \times \mathbb{P}^{1}$ and computed the Hilbert function for an arbitrary multiplicity except for a finite region in the space of multi-indices, see Carlini et al. (2017).

Remark 3.3 Once again, by using Terracini's Lemma, the computation of the Hilbert function of double points in generic position in a multi-projective space is related to the dimension of the secant varieties of Segre and Segre-Veronese varieties; see e.g. Catalisano et al. (2005), Catalisano et al. (2005), Catalisano et al. (2011). Moreover, as explained in Remark 3.2, the Hilbert functions of other types of 0-dimensional schemes in multi-projective spaces can be used to compute the dimensions of the secant varieties of other varieties such as tangential varieties of Segre and Segre-Veronese varieties; see e.g. Catalisano and Oneto (2018).

Remark 3.4 One of the major differences between the study of the Hilbert function of sets of (fat) points in projective spaces and the study of the Hilbert function of sets of (fat) points in multiprojetive spaces is that, in the latter setting, the coordinate ring of a collection of points is not always Cohen-Macaulay. There is an extensive literature studying the Hilbert functions and other algebraic properties of ideals of arithmetically Cohen-Macaulay (aCM) points in multi-projective spaces; see e.g. Guardo and Van Tuyl (2015). However, these sets of points in multi-projective spaces are very special and, in some sense, Problem K considers the opposite situation, where the points are assumed to be generic.

\subsection{Symbolic Powers vs. Ordinary Powers}

As we mentioned above, if $I$ is the ideal defining a set $X$ of points, then the $m$-th symbolic power of $I$ is the ideal of polynomials vanishing of order at least $m-1$ at all points in $X$. In other words, it is the space of hypersurfaces which are singular at all points of $X$ up to order $m-1$. For this reason, symbolic powers are interesting from a geometrical point of view, but they are more difficult to study compared to the ordinary powers which carry less geometrical information. Hence, it is important to find relations between these two. Observe that the inclusion $I^{m} \subset I^{(m)}$ is trivial.

Containment problems between the ordinary and the symbolic powers of ideals of points have been studied in substantial detail. One particularly interesting question is to understand for which positive integers $m$ and $r$, we have $I^{(m)} \subset I^{r}$. A very important result in this direction is the statement that, for any ideal $I$ of reduced points in $\mathbb{P}^{n}$ and any $r>1$, we have $I^{(n r)} \subset I^{r}$. This theorem was proven in Ein et al. (2001) by L. Ein, Lazersfeld and Smith in characteristic 0 and by Hochster and Huneke in positive characteristic, see Hochster and Huneke (2002). At present, the important question is whether the bound in the latter statement is sharp. In Dumnicki et al. (2013), Dumicki, Szemberg and Tutaj-Gasińska provided the first example of a configuration of points 
such that $I^{(3)} \not \subset I^{2}$. (We refer to Szemberg and Szpond (2017) for a complete account of this topic.)

In the recent paper Galetto et al. (2016), Galetto, Geramita, Shin and Van Tuyl defined the $m$-th symbolic defect of an ideal as the number of minimal generators of the quotient ideal $I^{(m)} / I^{m}$. If $I$ defines a set of general points in projective space, it was already known that $I^{(m)}=I^{m}$ if and only if $I$ is a complete intersection. Additionally, in [Galetto et al. (2016), Theorem 6.3] the authors characterize all cases of $s$ points in $\mathbb{P}^{2}$ having the 2-nd symbolic defect equal to 1 . These cases are exactly $s=3,5,7,8$.

Problem $\mathrm{L}$ For the ideal I of s general points in $\mathbb{P}^{n-1}$, what is the difference between the Hilbert series of the $m$-th symbolic power and the $m$-th ordinary power?

\section{Miscellanea}

\subsection{Hilbert Series of Numerical Semigroup Rings}

Let $\mathcal{S}=\left\langle s_{1}, \ldots, s_{n}\right\rangle$ be a numerical semigroup, i.e. $\mathcal{S}$ consists of all linear combinations with non-negative integer coefficients of the positive integers $s_{i}$. Let $\mathbb{C}[\mathcal{S}]=\mathbb{C}\left[x^{s_{1}}, \ldots, x^{s_{n}}\right]$ be the semigroup ring. The ring $\mathbb{C}[\mathcal{S}]$ is the image of $\phi: \mathbb{C}\left[t_{1}, \ldots, t_{n}\right] \rightarrow \mathbb{C}[x]$, where $\phi\left(t_{i}\right)=x^{s_{i}}$. If we let $\operatorname{deg}\left(t_{i}\right)=s_{i}$, the map will be graded, and we can define the Hilbert series of $\mathbb{C}[\mathcal{S}]$ as $\sum_{i=0}^{\infty} \operatorname{dim}_{\mathbb{C}} \mathbb{C}[\mathcal{S}]_{i} t^{i}$. If $\mathbb{C}[\mathcal{S}]$ happens to be a complete intersection, $\mathbb{C}[\mathcal{S}]=\mathbb{C}\left[t_{1}, \ldots, t_{n}\right] /\left(r_{1}, \ldots, r_{n-1}\right)$, where the $r_{i}$ 's are homogeneous relations, and the Hilbert series is $\prod_{i=1}^{n-1}\left(1-t^{\operatorname{deg} r_{i}}\right) / \prod_{i=1}^{n}(1-$ $\left.t^{s_{i}}\right)$. Thus the numerator has all its roots on the unit circle. For any numerical semigroup the Hilbert series is of the form $p(t) / \prod_{i=1}^{n}\left(1-t^{s_{i}}\right)$, where $p(t)$ is a polynomial with integer coefficients. A semigroup is called cyclotomic if the polynomial $p(t)$ has all its roots in the unit circle (which in fact implies that they lie on the unit circle if they are non-zero) (more information about the following conjecture can be found in Ciolan et al. (2016)).

Conjecture $4.1 \mathcal{S}$ is cyclotomic if and only if $\mathbb{C}(\mathcal{S})$ is a complete intersection.

\subsection{Non-Negative Forms}

The next circle of problems is related to the celebrated article Hilbert (1888a) of Hilbert and to a number of results formulated in Choi et al. (1980).

Denote by $P_{n, m}$ the set of all non-negative real forms, i.e., real homogeneous polynomials of (an even) degree $m$ in $n$ variables which never attain negative values; denote by $\Sigma_{n . m} \subseteq P_{n, m}$ the subset of non-negative forms which can be represented as sums of squares of real forms of degree $\frac{n}{2}$. (In Hilbert (1888a) Hilbert proved that $\Delta_{n, m}=P_{n, m} \backslash \Sigma_{n, m}$ is non-empty unless the pair $(n, m)$ is of the form $(n, 2),(2, m)$ or $(3,4)$.) Important qualitative characterization of $\Delta_{n, m}$ was obtained 6 years ago by G. Blekherman, see Blekherman (2012). Following the original Blekherman (2012), many more results dealing with $\Delta_{n, m}$ for some special situations and/or special values of $n$ and $m$ were obtained during the last 5 years. One natural question in this area is 
to find some properties of a non-negatie multivariate polynomial which will ensure that it belongs to the difference $\Delta_{n, m}$. The next problem is pointing in this direction.

Namely, if $\mathcal{Z}(p)$ stands for the real zero locus of a real form $p$, denote by $B_{n, m}$ (resp. $B_{n, m}^{\prime}$ ) the supremum of $|\mathcal{Z}(p)|$ over $p \in P_{n, m}$ such that $|\mathcal{Z}(p)|<\infty$ (resp. over $p \in \Sigma_{n, m}$ such that $\left.|\mathcal{Z}(p)|<\infty\right)$. In other words, $B(n, m)$ is the supremum of the number of zeros of non-degenerate forms under the assumption that all these roots are isolated (and similarly for $B_{n, m}^{\prime}$ ). Obviously, $B_{n, m}^{\prime} \leq B_{n, m}$.

The following basic question was posed in Choi et al. (1980).

Problem $\mathrm{M}$ Are $B_{n, m}$ and $B_{n, m}^{\prime}$ finite for any pair $(n, m)$ with even $n$ ?

In Choi et al. (1980) it was shown that the answer to this problem is positive for $m=2,3$ and for the pair $(4,4)$. Relatively recently, in Cartwright and Sturmfels (2013) the following upper bound for $B_{n, m}$ was established

$$
B_{n, m} \leq 2 \frac{(m-1)^{n+1}-1}{m-2} \text {. }
$$

However this bound can not be sharp, as shown in Kozhasov (2017). It seems however that the above finiteness follows from the classical Petrovski-Oleinik inequalities, see Petrovskii and Oleinik (1949). According to I. Itenberg (private communication) Petrovksi's estimate using the middle Hodge number of the complex zero locus a non-negative polynomial seems to give a better upper bound than the above one.

On the other hand, in case of $B_{n, m}^{\prime}$, the following guess seems quite plausible and is proven in the original paper Choi et al. (1980) for $m=3$.

Conjecture 4.2 For any given pair $(n, m)$ with even $n, B_{n, m}^{\prime}=\left(\frac{m}{2}\right)^{n-1}$.

For $B_{n, m}$, no similar guess is known, but some intriguing information is available in the case $n=3$, see Choi et al. (1980). The following problem is also related to the classical Petrovski-Oleinik upper bound on the number of real ovals of real plane algebraic curves.

Problem N Determine $\lim _{m \rightarrow \infty} \frac{B_{3, m}}{m^{2}}$.

The latter limit exists and lies in the interval $\left[\frac{5}{18}, \frac{1}{2}\right]$, see Choi et al. (1980). According to I. Itenberg (private communication), he jointly with A. Degtyarev and E. Brugalle has an improvement of the left endpoint of the latter interval.

\subsection{Polynomial Generation}

Let $p$ be a prime number and let $\mathbb{F}_{p}$ denote the field with $p$ elements. Consider the two maps

$$
\begin{aligned}
& \phi: \mathbb{F}_{p}\left[x_{1}, \ldots, x_{n}\right] \rightarrow \mathbb{F}_{p}\left[x_{1}, \ldots, x_{n}\right], f \mapsto \sum_{a \in \mathcal{Z}(f)} x^{a}, \\
& \psi: \mathbb{F}_{p}\left[x_{1}, \ldots, x_{n}\right] \rightarrow \mathbb{F}_{p}\left[x_{1}, \ldots, x_{n}\right], f \mapsto \sum_{a \in \mathbb{F}_{p}^{n}} f(a) x^{a} .
\end{aligned}
$$


Here $x^{a}:=x_{1}^{a_{1}} \cdots x_{n}^{a_{n}}$, where each $a_{i}$ is regarded as an integer, and $\mathcal{Z}(f)$ is the zero locus of $f$ in $\mathbb{F}_{p}^{n}$, i.e., $\mathcal{Z}(f):=\left\{a \in \mathbb{F}_{p}^{n} \mid f(a)=0\right\}$. When $p=2$, the map $\phi$ is a bijection on the vector space of polynomials of degree at most one in each variable, and $\phi^{4}(f)=f$, see Lundqvist (2015).

The map $\psi$, suggested by Boij, is a linear bijective map on the vector space of polynomials with degree at most $p-1$ in each variable, and when $p=2$, these two maps are closely related in the sense that $\phi(f)=\psi(f)+\sum_{a \in \mathbb{F}_{2}^{n}} x^{a}$.

Consider now the case $n=1$ and $p>2$. The map $\phi$ is no longer a bijection, but by a dimension argument, the sequence $\phi(f), \phi^{2}(f), \ldots$ will eventually become periodic. It is an easy exercise to show that

$$
0 \mapsto 1+x+\cdots+x^{p-1} \mapsto x \mapsto 1 \mapsto 0,
$$

so for each $p$, there exists a period of length four. By exhaustive computer search we have shown that when $p \leq 17$, this is the only period, i.e., $\phi(f)^{d(f)}=0$ for some $d(f)$, eventually giving the period (7). For $p=71$, we have found a period of length two;

$$
1+x^{63} \mapsto x^{23}+x^{26}+x^{34}+x^{39}+x^{41}+x^{51}+x^{70} \mapsto 1+x^{63} .
$$

One can easily show that the length of the period is always an even number, but it is not clear which even numbers that can occur as lengths of periods. So far, we are only aware of periods of length two and four.

Problem 0 For $n=1$ and given $p$, what are the (lengths of the) possible periods of $\phi ?$

Let us now turn to the map $\psi$ and the case $n=1$. For $p=3, \psi^{8}(f)=f$ for all polynomials $f$ in $\mathbb{F}_{3}[x]$ of degree at most two, as can be checked by hand. For $p=5$, a computer calculation shows that the least $i$ such that $\psi^{i}=\operatorname{Id}$ on the space of polynomials of degree at most four, is equal to 124 . With brute force, we also managed to determine to corresponding number for $p=7$ to 1368 , and for $p=11$ to 32129475 .

Problem $\mathbf{P}$ For $n=1$ and given $p$, find the minimal positive integer $i$ such that $\psi^{i}$ is the identity map on the space of polynomials of degree at most $p-1$.

\subsection{Exterior Algebras}

Denote by $E$ the exterior algebra on $n$ generators over $\mathbb{C}$. Like the polynomial ring, the algebra $E$ is naturally graded. Since the square of a generator in $E$ is zero, one has $E=E_{0} \oplus E_{1} \oplus \cdots \oplus E_{n}$, and the Hilbert series of $E$ is equal to $(1+t)^{n}$. Notice that this is the same Hilbert series as for the squarefree algebra $S /\left(x_{1}^{2}, \ldots, x_{n}^{2}\right)$. A natural problem is to find an analog to Fröberg's conjecture for quotients of exterior algebras with ideals generated by generic forms. A first guess would be that the Hilbert series of $E /\left(f_{1}, \ldots, f_{r}\right)$, each $f_{i}$ a generic form of degree $d_{i}$, is equal to

$$
\left[(1+t)^{n} \prod_{i=1, \ldots, r}\left(1-t^{d_{i}}\right)\right]_{+} .
$$


Moreno-Socías and Snellman (2002) showed that this is the case when $r=1$ and $d_{1}$ is even. However, since $f^{2}=0$ when $f$ has odd degree in $E$, it holds that $(f) \subseteq \operatorname{Ann}(f)$. Thus, the series cannot be equal to $\left[(1+t)^{n}\left(1-t^{d}\right)\right]_{+}$when $f$ has odd degree equal to $d$.

The annihilator ideal Ann $(f)$ shows some unexpected behavior. Indeed, when $(n, d)=(9,3)$, the map induced by multiplication be a generic cubic form from $E_{3}$ (of dimension $\left(\begin{array}{l}9 \\ 3\end{array}\right)$ ) to $E_{6}$ (of dimension $\left(\begin{array}{l}9 \\ 6\end{array}\right)=\left(\begin{array}{l}9 \\ 3\end{array}\right)$ ) has a kernel of dimension four, see Lundqvist and Nicklasson (2018), while a one-dimensional kernel is what one would expect.

However, we find it natural to believe that the graded pieces of $\operatorname{Ann}(f)$ behaves as expected in low degrees. The next problem is inspired by [Lundqvist and Nicklasson (2018), Question 1].

Problem Q Let $f$ be a generic form of odd degree $d$ in E. Is it true that $(\operatorname{Ann}(f))_{i}=$ $(f)_{i}$ for $i<(n-d) / 2$ ?

A lower bound for the series of $E /(f)$ is given in Lundqvist and Nicklasson (2018). It is also shown that the lower bound agrees with the generic series is some special cases. To show that the lower bound is equal to the Hilbert series for $E /(f)$, with $f$ generic, it is enough to find an explicit form such that the Hilbert series of the corresponding quotient is equal to the lower bound. This was the method of proof used by Moreno-Socías and Snellman in the even case.

But while the form used in the even degree case has a simple structure - it is the sum of all monomials - there is at the moment no good candidate to use in the odd case. We are surprised over the difficulty of the problem.

Problem $\mathbf{R}$ For each $n$ and odd $d$, find a form $f$ of degree $d$ such that $E /(f)$ has the minimal series as given in Lundqvist and Nicklasson (2018).

We now turn to the problem of deciding the Hilbert series of the quotient of the ideal generated by two generic quadratic forms. One would expect that $E /(f, g)$ should have series equal to $\left[(1+t)^{n}\left(1-t^{2}\right)^{2}\right]_{+}$, but this is not true. Fröberg and Löfwall (2002) showed by a brute force calculation that when $n=5$, the generic series is equal to $\left[(1+t)^{5}\left(1-t^{2}\right)^{2}\right]_{+}+t^{3}$. In Crispin Quiñonez et al. (2018), the connection between pairs of quadratic forms and matrix pencils was used to show that the failure for $n=5$ is just the top of the iceberg.

We finish with the following conjecture from Crispin Quiñonez et al. (2018), which surprisingly connects the question about the Hilbert series of quotient of the exterior algebra with quotients of the polynomial ring.

Conjecture 4.3 Let $f$ and $g$ be generic quadratic forms in $E$ and let $\ell_{1}$ and $\ell_{2}$ be two generic linear forms in $S$. Then the Hilbert series of $E /(f, g)$ is equal to the Hilbert series of $S /\left(x_{1}^{2}, \ldots, x_{n}^{2}, \ell_{1}^{2}, \ell_{2}^{2}\right)$ and is given by $1+a(n, 1) t+a(n, 2) t^{2}+$ $\cdots+a(n, s) t^{s}+\cdots$, where $a(n, s)$ is the number of lattice paths inside the rectangle $(n+2-2 s) \times(n+2)$ starting from the bottom-left corner and ending at the top-right corner by using only moves of two types: either $(x, y) \rightarrow(x+1, y+1)$ or $(x-$ $1, y+1)$. 
Acknowledgements The authors want to thank all the participants of the problem-solving seminar at Stockholm University for their contributions and patience. The third author acknowledges financial support from the Spanish Ministry of Economy and Competitiveness, through the María de Maeztu Programme for Units of Excellence in R\&D (MDM-2014- 0445). The authors are sincerely grateful to Dr. Kh. Khozhasov for pointing out references Cartwright and Sturmfels (2013), Kozhasov (2017) and to Prof. I. Itenberg for discussions of the problems in Section 4.2.

Open Access This article is distributed under the terms of the Creative Commons Attribution 4.0 International License (http://creativecommons.org/licenses/by/4.0/), which permits unrestricted use, distribution, and reproduction in any medium, provided you give appropriate credit to the original author(s) and the source, provide a link to the Creative Commons license, and indicate if changes were made.

\section{References}

Abbott, J., Bigatti, A.M.: CoCoALib: a C++ library for doing Computations in Commutative Algebra. http://cocoa.dima.unige.it/cocoalib (2014)

Abo, H.: Varieties of completely decomposable forms and their secants. J. Algebra 403, 135-153 (2014)

Abo, H., Vannieuwenhoven, N.: Most secant varieties of tangential varieties to Veronese varieties are nondefective. Trans. Am. Math. Soc. 370(1), 393-420 (2018)

Alexander, J., Hirschowitz, A.: Polynomial interpolation in several variables. J. Algebra Geom. 4, 201-222 (1995)

Anick, D.: Thin algebras of embedding dimension three. J. Algebra 100, 235-259 (1986)

Ardila, F., Postnikov, A.: Combinatorics and geometry of power ideals. Trans. Am. Math. Soc. 362(8), 4357-4384 (2010)

Ardila, F., Postnikov, A.: Correction to "Combinatorics and geometry of power ideals": two counterexamples for power ideals of hyperplane arrangements. Trans. Am. Math. Soc. 367(5), 3759-3762 (2015)

Arrondo, E., Bernardi, A.: On the variety parameterizing completely decomposable polynomials. J. Pure Appl. Algebra 215, 201-220 (2011)

Aubry, M.: Série de Hilbert d'une algèbre de polynômes quotient. J. Algebra 176, 392-416 (1995)

Blekherman, Grigoriy: Nonnegative polynomials and sums of squares. J. Am. Math. Soc 25(3), 617-635 (2012)

Bernardi, A., Blekherman, G., Ottaviani. G.: On real typical ranks. Boll. Unione Mat. Ital. https://doi.org/ 10.1007/s40574-017-0134-0 (2017)

Bernardi, A., Catalisano, M.V., Gimigliano, A., Ida, M.: Secant varieties to osculating varieties of Veronese embeddings of $\mathbb{P}^{n}$. J. Algebra 321(3), 982-1004 (2009)

Ballico, E.: On the secant varieties to the tangent developable of a Veronese variety. J. Algebra 288, 279-286 (2005)

Ballico, E., De Paris, A.: Generic power sum decompositions and bounds for the Waring rank. Discrete Comput. Geom. 57(4), 896-914 (2017)

Blekherman, G.: Typical real ranks of binary forms. Found. Comput. Math. 15(3), 793-798 (2015)

Blekherman, G., Teitler, Z.: On maximum, typical, and generic ranks. Mathematische Annalen 362(3-4), 1021-1031 (2015)

Bodin, A., Car, M.: Waring's problem for polynomials in two variables. Proc. Am. Math. Soc. 141(5), 1577-1589 (2013)

Boij, M., Fröberg, R., Lundqvist, S.: Powers of generic ideals and the weak Lefschetz property for powers of monomial complete intersections. J. Algebra 495, 1-14 (2018)

Brambilla, M.C., Ottaviani, G.: On the Alexander-Hirschowitz theorem. J. Pure Appl. Algebra 212, 1229_ 1251 (2008)

Brenner, H., Kaid, A.: A note on the weak Lefschetz property of monomial complete intersections in positive characteristic. Collect. Math. 62(1), 85-93 (2011)

Car, M.: New bounds on some parameters in the Waring problem for polynomials over a finite field. Contemp. Math. 461, 59-77 (2008)

Car, M., Gallardo, L.: Sums of cubes of polynomials. Acta Arith. 112, 41-50 (2004)

Carlini, E., Catalisano, M.V., Geramita, A.V.: The solution to the Waring problem for monomials and the sum of coprime monomials. J. Algebra 370, 5-14 (2012) 
Carlini, E., Catalisano M.V., Oneto A.: On the Hilbert function of general fat points in $\mathbb{P}^{1} \times \mathbb{P}^{1}$, arXiv preprint arXiv:1711.06193 (2017)

Carlini, E., Kummer, M., Oneto, A., Ventura, E.: On the real rank of monomials. Math. Z. 286, 571-577 (2017)

Carlini, E., Oneto, A.: Monomials as sum of k-th powers of forms. Commun. Algebra 43, 650-658 (2015)

Cartwright, D., Sturmfels, B.: The number of eigenvalues of a tensor. Linear Algebra Appl. 438, 942-952 (2013)

Catalisano, M.V., Chiantini, L., Geramita, A.V., Oneto, A.: Waring-like decompositions of polynomials, 1. Linear Algebra Appl. 533, 311-325 (2017)

Catalisano, M.V., Geramita, A.V., Gimigliano, A.: On the secant varieties to the tangential varieties of a Veronesean. Proc. Am. Math. Soc. 130, 975-985 (2002)

Catalisano, M.V., Geramita, A.V., Gimigliano, A.: Higher secant varieties of the Segre varieties $\mathbb{P}^{1} \times \ldots \times \mathbb{P}^{1}$. J. Pure Appl. Algebra 201(1), 367-380 (2005)

Catalisano, M.V., Geramita, A.V., Gimigliano A.: Higher secant varieties of Segre-Veronese varieties, Projective varieties with unexpected properties, 81-107 (2005)

Catalisano, M.V., Geramita, A.V., Gimigliano, A.: Secant varieties of $\mathbb{P}^{1} \times \cdots \times \mathbb{P}^{1}$ (n-times) are NOT defective for $n \geq 5$. J. Algebraic Geom. 20, 295-327 (2011)

Catalisano, M.V., Geramita, A.V., Gimigliano, A., Harbourne, B., Migliore, J., Nagel, U., Shin, Y.S.: Secant varieties of the varieties of reducible hypersurfaces in $\mathbb{P}^{n}$, arXiv: 1502.00167 (2015)

Catalisano, M.V., Oneto, A.: Tangential varieties of Segre-Veronese surfaces are never defective, arXiv:1805.11964 (2018)

Causa, A., Re, R.: On the maximum rank of a real binary form. Annali di Matematica Pura ed Applicata 190(1), 55-59 (2011)

Chandler, K.: The geometric interpretation of Fröberg-Iarrobino conjectures on infinitesimal neighbourhoods of points in projective space. J. Algebra 286(2), 421-455 (2005)

Chiantini, L., Ottaviani, G., Vannieuwenhoven, N.: Effective criteria for specific identifiability of tensors and forms. SIAM J. Matrix Anal. Appl. 38(2), 656-681 (2017)

Choi, M.-D., Lam, T.-Y., Reznick, B.: Real Zeros of Positive Semidefinite Forms. I. Math. Z. 171, 1-26 (1980)

Ciolan, A., Garcia-Sanchez, P., Moree, P.: Cyclotomic numerical semigroups. SIAM J. Discrete Math. 30(2), 650-668 (2016)

Comon, P., Ottaviani, G.: On the typical rank of real binary forms. Linear Multilinear Algebra 60(6), 657-667 (2012)

Crispin Quiñonez, V., Lundqvist, S., Nenashev, G.: On ideals generated by two generic quadratic forms in the exterior algebra, arXiv:1803.08918 (2018)

De Paris, A.: Every ternary quintic is a sum of ten fifth powers, International Journal of Algebra and Computation (2015)

Decker, W., Greuel, G.-M., Pfister, G., Schönemann, H.: Singular 4-1-1-a computer algebra system for polynomial computations, http://www.singular.uni-kl.de (2018)

Dumnicki, M., Szemberg, T., Tutaj-Gasińska, H.: Counterexamples to the ${ }^{(3)} \subset I^{2}$ containment. J. Algebra 393, 24-29 (2013)

Ein, L., Lazarsfeld, R., Smith, K.: Uniform bounds and symbolic powers on smooth varieties. Invent. Math. 144, 241-252 (2001)

Fröberg, R.: An inequality for Hilbert series. Math. Scand. 56, 117-144 (1985)

Fröberg, R., Hollman, J.: Hilbert series for ideals generated by generic forms. J. Symb. Comp. 17, 149-157 (1994)

Fröberg, R., Lundqvist, S.: Questions and conjectures on extremal Hilbert series. Revista de la Unión Matemática Argentina. 59(2), 415-429 (2018)

Fröberg, R., Löfwall, C.: On Hilbert series for commutative and noncommutative graded algebras. J. Pure Appl. Algebra 76, 33-38 (1990)

Fröberg, R., Löfwall, C.: Koszul homology and Lie algebras with application to generic forms and points. Homol Homotopy Appl. 4, 227-258 (2002)

Fröberg, R., Ottaviani, G., Shapiro, B.: On the Waring problem for polynomial rings. PNAS 109(15), 5600-5602 (2012)

Galetto, F., Geramita, A.V., Shin, Y.S., Van Tuyl, A.: The symbolic defect of an ideal. arXiv:1610.00176 (2016)

Gallardo, L.: On the restricted Waring problem over $\mathbb{F}_{2^{n}}[t]$. Acta Arith. 42, 109-113 (2000) 
Gallardo, L., Vaserstein, L.: The strict Waring problem for polynomial rings. J. Number Theory 128, 29632972 (2008)

Galuppi, F., Mella, M. : Identifiability of homogeneous polynomials and Cremona transformations, J für die reine und angewandte Mathematik. https://doi.org/10.1515/crelle-2017-0043 (2017)

Geramita, A.V.: Waring's Problem for Forms: inverse systems of fat points, secant varieties and Gorenstein algebras. Queen's Papers Pure Appl. Math. 105(2), 1-129 (1996)

Geramita, A.V., Orecchia, F.: On the Cohen-Macaulay type of s lines in $\mathbb{A}^{n+1}$. J. Algebra 70(1), 116-140 (1981)

Geramita, A.V., Schenck, H.: Fat points, inverse systems, and piecewise polynomial functions. J. Algebra 204(1), 116-128 (1998)

Gimigliano, A.: On linear systems of plane curves, Ph. D. thesis, Queen's University, Kingston, Ontario (1987)

Grayson, D.R., Stillman, M.E.: Macaulay2, a software system for research in algebraic geometry. http:// www.math.uiuc.edu/Macaulay2/ (2002)

Guardo, E., Van Tuyl, A.: Aritmetically Cohen-Macaulay sets of points in $\mathbb{P}^{1} \times \mathbb{P}^{1}$, SpringerBriefs in Mathematics (2015)

Harbourne, B.: The geometry of rational surfaces and Hilbert functions of points in the plane. Can. Math. Soc. Conf. Proc. 6, 95-111 (1986)

Harbourne, B.: Problems, progress: a survey on fat points in P2, in Zero Dimensional Schemes and Applications, Naples, : 85-132. Queen's Papers in Pure and Appl, Mat (2000)

Harima, T., Maeno, T., Morita, H., Numata, Y., Wachi, A., Watanabe, J.: The Lefschetz properties. Lecture Notes in Mathematics, vol. 2080. Springer, Heidelberg (2013)

Harima, T., Migliore, J., Nagel, U., Watanabe, J.: The weak and strong Lefschetz properties for artinian $K$-algebras. J. Algebra 262, 99-126 (2003)

Herzog, J., Popescu, D. The strong Lefschetz property and simple extensions, arXiv:math/0506537 (2005)

Hilbert, D.: Über die Darstellung definiter Formen als Summe von Formenquadraten. Math. Ann. 32(3), 342-350 (1888a)

Hilbert, D.: Letter adressée à M. Hermite, Gesam. Abh. vol II, 148-153 (1888b)

Hirschowitz, A.: Une conjecture pour la cohomologie des diviseurs sur les surfaces rationelles geénériques. J. Reine Angew. Math. 397, 208-213 (1989)

Hochster, M., Huneke, C.: Comparison of symbolic and ordinary powers of ideals. Invent. Math. 147, 349-369 (2002)

Hochster, M., Laksov, D.: The linear syzygies of generic forms. Commun. Algebra 15, 227-234 (1987)

Iarrobino, A.: Inverse system of a symbolic power III. Thin algebras and fat points. Compositio Math. 108, 319-356 (1997)

Iarrobino, A., Kanev, V.: Power sums, Gorenstein algebras, and determinantal loci. Springer, New York (2006)

Kleppe, J.: Representing a homogeneous polynomial as a sum of powers of linear forms, Master Thesis, University of Oslo (1999)

Kozhasov, K.H.: On fully real eigenconfigurations of tensors. SIAM. J. Appl. Algebra Geometry 2(2), 339-347 (2017)

Jelisiejew, J.: An upper bound for the Waring rank of a form. Archiv der Mathematik 102(4), 329-336 (2014)

Kustin, A., Vraciu, A.: The weak Lefschetz property for monomial complete intersections in positive characteristic. Trans. Am. Math. Soc. 366, 4571-4601 (2014)

Landsberg, J.M.: Tensors: geometry and applications, vol. 128. American Mathematical Society, Providence, RI (2012)

Landsberg, J.M., Teitler, Z.: On the ranks and border ranks of symmetric tensors. Found. Comput. Math. 10(3), 339-366 (2010)

Le, T.H., Sorber, L., Van Barel, M.: The Pythagoras number of real sum of squares polynomials and sum of square magnitudes of polynomials. Calcolo 50(4), 283-303 (2013)

Liu, Y.-R., Wooley, T.D.: Waring's problem in function fields. J. Reine Angew. Math. 638, 1-67 (2010)

Lundqvist, S.: Boolean ideals and their varieties. J. Pure Appl. Algebra 219(5), 4521-4540 (2015)

Lundqvist, S., Nicklasson, L. On the structure of monomial complete intersections in positive characteristic, arXiv:1604.06820 (2016)

Lundqvist, S., Nicklasson, L.: On generic principal ideals in the exterior algebra, arXiv:1803.03563 (2018) 
Lundqvist, S., Oneto, A., Reznick, B., Shapiro, B.: On generic and maximal k-ranks of binary forms, arXiv:1711.05014 (2017)

Massarenti, A., Mella, M.: Birational aspects of the geometry of varieties of sums of powers. Adv. Math. 243, 187-202 (2013)

Michałek, M., Moon, H., Sturmfels, B., Ventura, E.: Real rank geometry of ternary forms. Annali di Matematica Pura ed Applicata 196(3), 1025-1054 (2017)

Michałek, M., Mirò-Roig, R.M.: Smooth monomial Togliatti systems of cubics. J. Combinatorial Theory Series A 143, 66-87 (2016)

Migliore, J., Mirò-Roig, R.M.: Ideals of general forms and the ubiquity of the weak Lefschetz property. J. Pure Appl. Algebra 102, 79-107 (2003)

Migliore, J., Nagel, U.: Survey article: a tour of the weak and strong Lefschetz properties. J. Commut. Algebra 5(3), 329-358 (2013)

Migliore, J., Nagel, U., Schenk, H.: The weak Lefschetz property for quotients by Quadratic Monomials, arXiv:1706.05058 (2017)

Moreno-Socías, G., Snellman, J.: Some conjectures about the Hilbert series of generic ideals in the exterior algebra. Homol. Homotopy Appl. 4, 409-426 (2002)

Nenashev, G.: A note on Fröbergs's conjecture for forms of equal degree. Comptes Rendus Mathematique 355(3), 272-276 (2017)

Nicklasson, L.: On the Hilbert series of ideals generated by generic forms. Commun. Algebra 45(8), 33903395 (2017)

Nicklasson, L.: The strong Lefschetz property of monomial complete intersections in two variables. Collect. Math. (2017). https://doi.org/10.1007/s13348-017-0209-3

Oneto, A.: Waring-type problems for polynomials, Doctoral Thesis in Mathematics, Stockholm University, Stockholm, Sweden (2016)

Palatini, F.: Sulla rappresentazione delle forme ternarie mediante la somma di potenze di forme lineari. Rom. Acc. L. Rend. 12, 378-384 (1903)

Palatini, F.: Sulle superficie algebriche i cui $S_{h}(h+1)$-seganti non riempiono lo spazio ambiente. Atti. Accad. Torino 41, 634-640 (1906)

Palatini, F.: Sulle varietá algebriche per le quali sono di dimensione minore dell'ordinario, senza riempire lo spazio ambiente, una o alcune delle varietá formate da spazi seganti. Atti. Accad. Torino 44, 362-374 (1909)

Petrovskii, I.G.; Oleinik, O.A.: On the topology of real algebraic surfaces (Russian) Izvestiya Akad. Nauk SSSR. Ser. Mat. 13, 389-402 (1949)

Reid, L., Roberts, L.G., Roitman, M.: On complete intersections and their Hilbert functions. Can. Math. Bull. 34, 525-535 (1991)

Reznick, B.: Some new canonical forms for polynomials. Pac. J. Math. 266(1), 185-220 (2013)

Reznick, B.: On the length of binary forms, Quadratic and Higher Degree Forms, (K. Alladi, M. Bhargava, D. Savitt, P. Tiep, eds.), Developments in Mathematics 31, 207-232, Springer New York (2013)

Richmond, H.W.: On canonical forms. Quart. J. Pure Appl. Math. 33, 967-984 (1904)

Scorza, G.: Determinazione delle varietá a tre dimensioni di $S \geq r, r \geq 7$, i cui $S_{3}$ tangenti si tagliano a due a due. Rend. Circ. Mat. Palermo 25, 193-204 (1908)

Shin, Y.: Secants to the variety of completely reducible forms and the Hilbert function of the union of star-configurations, J. Algebra Appl. 11 (2012)

Segre, B.: The non-singular cubic surfaces. Oxford University Press, Oxford (1942)

Segre, B.: Alcune questioni su insiemi finiti di punti in Geometria Algebrica. Atti del Convegno Internaz. di Geom. Alg, Torino (1961)

Stanley, R.: Hilbert functions of graded algebras. Adv. Math. 28, 57-83 (1978)

Stanley, R.: Weyl groups, the hard Lefschetz theorem, and the Sperner property. SIAM J. Algebraic Discrete Methods 1(2), 168-184 (1980)

Sylvester, J.J.: On a remarkable discovery in the theory of canonical forms and of hyperdeterminants, originally in Philosophical Magazine, vol. I, 1851; pp. 265-283 in Paper 41 in Mathematical Papers, Vol. 1, Chelsea, New York, (1973). Originally published by Cambridge University Press in 1904

Szemberg, T., Szpond, J.: On the containment problem. Rendiconti del Circolo Matematico di Palermo Series 2 66(2), 233-245 (2017)

Terracini, A.: Sulle $V_{k}$ per cui la varietá degli $S_{h}(h+1)$-seganti ha dimensione minore dell' ordinario. Rend. Circ. Mat. Palermo 31, 392-396 (1911)

Torrance, D.A. Generic forms of low Chow rank. J. Algebra. Appl. 16(3), 1750047 (2017) 
Vraciu, A.: On the degrees of relations on $x_{1}^{d_{1}}, \ldots, x_{n}^{d_{n}},\left(x_{1}+\cdots+x_{n}\right)^{d_{n}+1}$ in positive characteristic. J. Algebra 423, 916-949 (2015)

Zak F.: Tangents and secants of algebraic varieties, Transl. Math. Monogr. 127, Am. Math. Soc., Providence, RI (1993) 